\title{
DETECTION OF SPECIAL CURVES VIA THE DOUBLE RESULTANT
}

\author{
ANTONI FERRAGUT ${ }^{1}$, JOHANNA D. GARCÍA-SALDAÑA ${ }^{2}$ AND ARMENGOL GASULL ${ }^{3}$
}

\begin{abstract}
We introduce several applications of the use of the double resultant through some examples of computation of different nature: special level sets of rational first integrals for rational discrete dynamical systems; remarkable values of rational first integrals of polynomial vector fields; bifurcation values in phase portraits of polynomial vector fields; and the different topologies of the offset of curves.
\end{abstract}

\section{Introduction. The double Resultant}

In different situations such as remarkable curves of rational first integrals, bifurcation values of phase portraits of discrete or continuous dynamical systems, or the study of the offset of curves, there are algebraic curves which are special in the sense that have singularities or are the boundary of different topological behaviors. In this paper we present a systematic approach to detect the special values that provide these kind of special curves.

Let $F_{b}(x, y) \in \mathbb{C}[x, y, b]$ be an irreducible polynomial, with $b$ a set of parameters. We write $F_{b}$ as a polynomial in $y$ :

$$
F_{b}(x, y)=a_{n} y^{n}+a_{n-1} y^{n-1}+a_{n-2} y^{n-2}+\ldots+a_{1} y+a_{0},
$$

where $a_{i}=a_{i}(x)=a_{i}(x, b) \in \mathbb{C}[x, b]$. If $a_{n} \neq 0$, then the discriminant of $F_{b}$ with respect to the variable $y$ is defined in the literature as

$$
\operatorname{disc}_{y}\left(F_{b}\right)=(-1)^{\frac{n(n-1)}{2}} \frac{1}{a_{n}} \operatorname{Res}\left(F_{b}, \frac{\partial F_{b}}{\partial y}, y\right),
$$

and this resultant can be computed as the determinant of the Sylvester matrix of dimension $2 n-1$, see $[9]$.

In this work, instead of the discriminant, we shall simply use Res $\left(F_{b}, \frac{\partial F_{b}}{\partial y}, y\right)$, because the coefficient $a_{n}$ is important to keep information on $F_{b}$ at infinity, see for instance Example 1 in next section. We will name the resultant of $F_{b}$ with respect to $y$ the polynomial in $x$ (and $b$ )

$$
\Delta_{y}\left(F_{b}\right)=\operatorname{Res}\left(F_{b}, \frac{\partial F_{b}}{\partial y}, y\right) .
$$

In the same way we define $\Delta_{x}\left(F_{b}\right)=\operatorname{Res}\left(F_{b}, \frac{\partial F_{b}}{\partial x}, x\right)$, the resultant of $F_{b}$ with respect to $x$. We also remark that, for simplicity, along all the work when computing resultants we shall remove scalar nonzero constants without any explicit mention.

We write $\Delta_{y, x}\left(F_{b}\right)=\Delta_{x}\left(\Delta_{y}\left(F_{b}\right)\right)$. We name this polynomial the double resultant of $F_{b}$ with respect to $y, x$. Analogously we can compute $\Delta_{x, y}\left(F_{b}\right)$. We notice that they only depend on the set of parameters $b$ and that in general $\Delta_{x, y}\left(F_{b}\right) \neq \Delta_{y, x}\left(F_{b}\right)$.

2010 Mathematics Subject Classification. 34C05, 34A34, 34C14, 14P25, 14Q05, 68U01.

Key words and phrases. resultant, remarkable curves, integrability, offset of curves.

The authors are partially supported by MINECO/FEDER MTM2013-40998-P grant. The second author is also supported by FONDECyT postdoctoral fellowship 3150131/2015. The third author is also supported by Generalitat de Catalunya grant 2014SGR568. 
We will see that these polynomials play an important role in the characterization of singularities of $\left\{F_{b}(x, y)=0\right\}$. Our first result adapts the approach of [14, App. II] using discriminants to our setting and it is a key point for our work.

Theorem 1. Let $F(x, y) \in \mathbb{C}[x, y]$ be a complex polynomial and assume that $F=0$ has a singular point. If $\operatorname{deg}_{y}(F)>1$ then $\Delta_{y, x}(F)=0$ and, similarly, if $\operatorname{deg}_{x}(F)>1$ then $\Delta_{x, y}(F)=0$.

Note that the hypotheses on the degrees of $F$ are necessary. Otherwise, consider for instance $F(x, y)=x y$. Clearly, $F=0$ has a singular point at $(0,0)$ and $\Delta_{y, x}(F)=$ $\Delta_{x, y}(F)=1$.

As a corollary of Theorem 1 we obtain the following result.

Corollary 2. Let $F_{b}(x, y)=0$ be a family of algebraic curves (1) such that $\operatorname{deg}_{x}\left(F_{b}\right)>1$ and $\operatorname{deg}_{y}\left(F_{b}\right)>1$. Then the values of $b$ for which $F_{b}(x, y)=0$ has some singular point in $\mathbb{C}^{2}$ are zeros of the polynomial

$$
\Delta^{2}\left(F_{b}\right):=\operatorname{gcd}\left(\Delta_{x, y}\left(F_{b}\right), \Delta_{y, x}\left(F_{b}\right)\right) .
$$

For simplicity we will also call the polynomial $\Delta^{2}\left(F_{b}\right)$ the double resultant of the family $F_{b}(x, y)$. We shall use the name special values to refer the values of $b$ such that $\Delta^{2}\left(F_{b}\right)=0$. We remark that the name special values or critical values is also used in the literature to name the values where the corresponding double discriminants vanish. These values also play a significative role in the characterization of singular curves and they are used in many applications, see for instance $[2,19,22]$.

The double resultants $\Delta_{x, y}\left(F_{b}\right)$ and $\Delta_{y, x}\left(F_{b}\right)$ may usually provide values that do not correspond to special values. This is the reason for which considering their greatest common divisor we keep only the values that vanish both.

When we try to obtain the special values for some $F_{b}$ such that either $\operatorname{deg}_{x}\left(F_{b}\right)=1$ or $\operatorname{deg}_{y}\left(F_{b}\right)=1$ we can no more use Corollary 2. In this situation, we propose to utilize the so called Tame maps as changes of variables, see for instance [24] for a definition. These changes increase the degree of $F_{b}$ and hence the corollary can be applied. As an example of the utility of this procedure see for instance Example 2 in Section 2 or Example 3 in Section 3.2.

The main objective in this paper is to show that Corollary 2 and a modification of it, that we will describe below, supply a very useful tool in several problems. Using them we shall provide examples of computation of several objects such as special level sets of rational first integrals of discrete dynamical systems; bifurcation values of polynomial vector fields; remarkable values of rational first integrals of planar vector fields; and special values in the study of the topology of offset curves. We dedicate one section to each of these applications.

We describe here two main differences between our approach and previous works dealing with similar questions, see for instance $[1,2,3,19,22]$. The first one is that we use resultants instead of discriminants. As we have already mentioned, the reason is that removing the coefficient $a_{n}$ may lead to loose information about some changes of $F_{b}=0$ near infinity. The second one is that we compute both double resultants and afterwards their greatest common divisor. This procedure allows to remove certain superfluous values of $b$ that are not really special values of $F_{b}$, see for instance the comments in Section 3.4.

We end this introduction with a small modification of the method suggested by Corollary 2 based on the common use of double discriminants in the literature. As usual, given a polynomial in one variable $p(x)$ we will denote by $\sqrt{p(x)}$ the new square free polynomial obtained keeping the product of all its irreducible factors powered to one. We introduce the polynomial

$$
\Delta_{*}^{2}\left(F_{b}\right):=\operatorname{gcd}\left(\Delta_{y}\left(\sqrt{\Delta_{x}\left(F_{b}\right)}\right), \Delta_{x}\left(\sqrt{\Delta_{y}\left(F_{b}\right)}\right)\right) .
$$


Notice for instance that if $\Delta_{x}\left(F_{b}\right)$ has some multiple factor then $\Delta_{y}\left(\Delta_{x}\left(F_{b}\right)\right)$ identically vanishes, while $\Delta_{y}\left(\sqrt{\Delta_{x}\left(F_{b}\right)}\right)$ does not. This new modified approach consists in computing $\Delta_{*}^{2}\left(F_{b}\right)$ instead of $\Delta^{2}\left(F_{b}\right)$ to obtain the set of possible special values for $F_{b}$. Although we have not been able to provide a full justification of why this modified approach works, in Section 4 we give some ideas of why this argument rules and no information is lost. We recently knew that the consideration of only the square free part of the first resultant has been also used in the context of cylindrical algebraic decomposition, see [18].

\section{Some examples ANd PRoof of Theorem 1}

We start with an example that clearly shows the advantage, in our setting, of using resultants instead of discriminants. Recall that in the classical definition of the discriminant, since we are considering $a_{n} \neq 0$ we divide the resultant by $a_{n}$. But for our purposes, the case $a_{n}=0$ is also relevant, since if it vanishes the degree of $F_{b}$ decreases, and then the behavior of $F_{b}=0$ might change.

Example 1. Let $F_{b}(x, y)=-x^{2}+b y^{2}-x+y$. If $b=1$ then $F_{b}=0$ is formed by two straight lines; if $b>0$ but $b \neq 1$, then we have an hyperbola; if $b<0$ then we have an ellipse; and if $b=0$ then we have a parabola. Hence the values $b=0,1$ are special.

The discriminants of $F_{b}$ are as follows:

$$
\begin{gathered}
\operatorname{disc}_{x}\left(F_{b}\right)=1+4 y+4 b y^{2}, \quad \operatorname{disc}_{y}\left(F_{b}\right)=1+4 b x+4 b x^{2}, \\
\operatorname{disc}_{y}\left(\operatorname{disc}_{x}\left(F_{b}\right)\right)=-16(b-1), \quad \operatorname{disc}_{x}\left(\operatorname{disc}_{y}\left(F_{b}\right)\right)=16 b(b-1) .
\end{gathered}
$$

We can compute the greatest common divisor of the two double discriminants to obtain $16(b-1)$. Note that the case $b=0$ is not considered in the double discriminant, although it is important as we have explained.

On the other hand, the double resultant is $\Delta^{2}\left(F_{b}\right)=b(b-1)$, and thus all the special values are being considered.

We would like to comment here that other authors (see [6]) use a different approach, based on the the so-called bivariate discriminants, for finding singular points in the projective space, unifying the study of finite and infinite singular points.

Proof of Theorem 1. Assume that $n=\operatorname{deg}_{y}(F)>1$. Then $F$ writes as

$$
F(x, y)=a_{n} y^{n}+a_{n-1} y^{n-1}+a_{n-2} y^{n-2}+\ldots+a_{1} y+a_{0} .
$$

Recall that $F$ has a singular point if there exists $\left(x_{0}, y_{0}\right) \in \mathbb{C}^{2}$ such that $F\left(x_{0}, y_{0}\right)=$ $\partial F\left(x_{0}, y_{0}\right) / \partial x=\partial F\left(x_{0}, y_{0}\right) / \partial y=0$. Without loss of generality we assume that $\left(x_{0}, y_{0}\right)=$ $(0,0)$. Then since it is singular it follows that $a_{0}(0)=a_{0}^{\prime}(0)=0$ and $a_{1}(0)=0$. Therefore, $a_{0}(x)=x^{2} b_{0}(x)$ and $a_{1}(x)=x b_{1}(x)$, with both $b_{i}$ also polynomials.

The Sylvester matrix $S:=S(x):=\operatorname{Res}\left(F, \frac{\partial F}{\partial y}, y\right)$ is

$$
S=\left(\begin{array}{cccccccc}
a_{n} & 0 & 0 & 0 & n a_{n} & 0 & 0 & 0 \\
a_{n-1} & a_{n} & 0 & 0 & (n-1) a_{n-1} & n a_{n} & 0 & 0 \\
a_{n-2} & a_{n-1} & \ddots & 0 & (n-2) a_{n-2} & (n-1) a_{n-1} & \ddots & 0 \\
\vdots & & \ddots & a_{n} & \vdots & & \ddots & n a_{n} \\
& \vdots & & a_{n-1} & & \vdots & & (n-1) a_{n-1} \\
a_{0} & & & & a_{1} & & & \vdots \\
0 & a_{0} & & \vdots & 0 & a_{1} & & \vdots \\
0 & 0 & \ddots & & 0 & 0 & \ddots & \\
0 & 0 & 0 & a_{0} & 0 & 0 & 0 & a_{1}
\end{array}\right) .
$$


Then, for $n>1$,

$$
\operatorname{det} S=(-1)^{n} a_{0} \operatorname{det}\left(S_{n-1}\right)+a_{1} \operatorname{det}\left(S_{2 n-1}\right),
$$

where $S_{j}$ denotes the $2 n-2$ matrix obtained from $S$ by removing the last row and the $j$-th column.

Note that the last row of $S_{2 n-1}$ only contains zeros, $a_{0}$ and $a_{1}$. Therefore, developing the determinant of this matrix from this row we get that each summand has either the factor $a_{0}$ or the factor $a_{1}^{2}$. In any case we get that $\operatorname{det}\left(S_{2 n-1}\right)=x B(x)$, for some polynomial $B$.

Hence, by using (3), we obtain that $\operatorname{det} S=x^{2} C(x)$ with $C$ another polynomial. This implies that $\Delta_{y}(F)$ has a double zero at $x=0$ and hence $\Delta_{y, x}^{2}(F)=0$.

The second assertion follows by interchanging $x$ and $y$.

The proof of Corollary 2 is straightforward using Theorem 1. To explain how we can proceed to improve the detection of special values with some of the degrees of $\operatorname{deg}_{y}\left(F_{b}\right)$ or $\operatorname{deg}_{x}\left(F_{b}\right)$ is one, we consider a simple example.

Example 2. Set $F_{b}(x, y)=x^{3}+b(x-y)-x y+1$. We notice that $F_{b}=0$ can be viewed as the graph $y=\left(1+b x+x^{3}\right) /(b+x)$, which has a vertical asymptote on $x=-b$. Moreover for a certain real value $b \approx 0.7549$ the denominator of this quotient divides the numerator and hence the graph is a parabola. Thus this value of $b$ is a special value. $F_{b}$ has no other special values.

Let us proceed with our method. We compute first the resultants of $F_{b}$ both respect to $x$ and $y$ :

$$
\Delta_{x}\left(F_{b}\right)=-4 b^{3}-27+6 b(2 b+9) y-3 b(9 b+4) y^{2}+4 y^{3}, \quad \Delta_{y}\left(F_{b}\right)=1 .
$$

We can also compute the double resultant

$$
\Delta_{x, y}\left(F_{b}\right)=(b-1)^{3}(b+1)^{3}\left(b^{3}+b^{2}-1\right) .
$$

This double resultant provides three real special values. On the other side, the resultant of $F_{b}$ with respect to $y$ is 1 and no information can be obtained from it. Hence, Corollary 2 gives no information and Theorem 1 reduces the set of special values to three. Hence using our approach we have found two values of $b$ which are not special.

As we will see, this solution can be sharpened using Tame changes of variables. The Tame change is a kind of Jacobian change of variables, that is a polynomial change of variables $X$ with an inverse that is also polynomial. It is such that $\operatorname{det}(D X)=1$ and has the form:

$$
(u, v)=X(x, y)=\left(\begin{array}{c}
x+p(y) \\
y
\end{array}\right),
$$

where $p \in \mathbb{C}[y]$. Clearly $\operatorname{det}(D X)=1$.

For our purposes we shall take $\operatorname{deg} p>1$. We consider the alternative function

$$
\bar{F}_{b}(x, y)=F_{b}(x+p(y), y) .
$$

We have:

(a) $\Delta_{x}\left(\bar{F}_{b}\right)=\Delta_{x}\left(F_{b}\right)$. This happens because the values of $y$ for which $F_{b}$ and $\bar{F}_{b}$ change are the same. After this consideration we notice that $\Delta_{x, y}\left(F_{b}\right)=\Delta_{x, y}\left(\bar{F}_{b}\right)$.

(b) $\Delta_{y}\left(\bar{F}_{b}\right) \neq 1=\Delta_{y}\left(F_{b}\right)$. This is because $\operatorname{deg}_{y} \bar{F}_{b}>1=\operatorname{deg}_{y} F_{b}$, since $\operatorname{deg} p>1$. Then $\Delta_{y, x}\left(\bar{F}_{b}\right)$ provides some values of the parameter $b$ that were not provided before.

Thus on one side the double resultant $\Delta^{2}\left(\bar{F}_{b}\right)$ has no more roots than $\Delta_{x, y}\left(F_{b}\right)$, and on the other side $\Delta^{2}\left(\bar{F}_{b}\right) \neq 1$, since $\Delta_{y, x}\left(\bar{F}_{b}\right) \neq 1$. Then we can compute the double resultant $\Delta^{2}\left(\bar{F}_{b}\right)$ after the Tame change of variable.

Example 2 (revisited). Consider again the polynomial $F_{b}(x, y)=x^{3}+b(x-y)-x y+1$. We apply to $F_{b}$ the change $x \mapsto x+y^{2}$ : we define $\bar{F}_{b}(x, y)=F_{b}\left(x+y^{2}, y\right)$. Now we compute 
the resultants of $\bar{F}_{b}$ with respect to both $x$ and $y: \Delta_{y}\left(\bar{F}_{b}\right)$ is a polynomial of degree 6 in $x$ and $\Delta_{x}\left(\bar{F}_{b}\right)=\Delta_{x}\left(F_{b}\right)$.

Now $\Delta_{x, y}\left(\bar{F}_{b}\right)=\Delta_{y, x}\left(F_{b}\right)$ and $\Delta_{y, x}\left(\bar{F}_{b}\right)=\left(b^{3}+b^{2}-1\right) P_{10}(b)^{2} P_{11}(b)^{3}$, where $P_{i}$ are polynomials of degree $i$ that do not vanish at $b= \pm 1$. The greatest common divisor of both resultants is $\Delta^{2}\left(\bar{F}_{b}\right)=b^{3}+b^{2}-1$. Hence we obtain only one real (special) value, as it was stated.

\section{Applications}

This section contains several applications of our approach for detecting special values of families of rational curves in different contexts. There are four subsections, the first devoted to discrete dynamical systems, next two to planar integrable vector fields and the last one to the offsets of curves.

3.1. Rationally integrable discrete dynamical systems. Our first example deals with the well-known Lyness difference equation $x_{n+2}=\left(a+x_{n+1}\right) / x_{n}$, see for instance $[5,16]$ and the references therein. It is clear that it can be studied through the iterates of its corresponding discrete dynamical system, which is given by the map

$$
G(x, y)=\left(y, \frac{a+y}{x}\right) .
$$

It is also well-known that the function

$$
V(x, y)=\frac{(1+x)(1+y)(a+x+y)}{x y},
$$

is a first integral for the map, i.e. $V(G(x, y))=V(x, y)$ for all $(x, y)$ for which the involved functions are well defined. Notice that for some few values of $h$ the invariant energy levels $\mathcal{V}_{a, h}=\{(x, y): V(x, y)=h\}$ are not elliptic curves. We will see how to determine these values with our approach.

Among other things this property is important for instance to study the existence of periodic points with rational coordinates, see for instance [16]. Recall that for the values $a, h \in \mathbb{Q}$ such that $\mathcal{V}_{a, h}$ is an elliptic curve, the celebrated Mazur's Torsion Theorem asserts that only periodic points of periods $1,2, \ldots, 9,10$ and 12 are possible. When $a$ and $h$ are such that $\mathcal{V}_{a, h}$ is not an elliptic curve other periods could appear. In fact, in these cases, the genus of the sets is zero and rational parameterizations can be used to study the periodic points.

Consider the sets $\mathcal{V}_{a, h}$, with $h \in \mathbb{R} \cup\{\infty\}$. Let us determine with our approach the values of $h$ for which they are not formed by elliptic curves. For convenience we write $V(x, y)=h$ as $F_{\lambda, \mu}(x, y)=0$,

$$
F_{\lambda, \mu}(x, y)=\lambda(1+x)(1+y)(a+x+y)+\mu x y,
$$

where $h=-\mu / \lambda$, with $\lambda, \mu \in \mathbb{R}$. Straightforward computations give

$$
\begin{aligned}
& \Delta_{x}\left(F_{\lambda, \mu}\right)=\lambda(1+y)\left((-1+a)^{2} \lambda^{2}+2 \lambda\left(-a \lambda+a^{2} \lambda+\mu+a \mu\right) y\right. \\
&\left.+\left(-2 \lambda^{2}+2 a \lambda^{2}+a^{2} \lambda^{2}+4 \lambda \mu+2 a \lambda \mu+\mu^{2}\right) y^{2}+2 \lambda(a \lambda+\mu) y^{3}+\lambda^{2} y^{4}\right), \\
& \Delta_{y}\left(F_{\lambda, \mu}\right)=\lambda(1+x)\left((-1+a)^{2} \lambda^{2}+2 \lambda\left(-a \lambda+a^{2} \lambda+\mu+a \mu\right) x\right. \\
&\left.\quad+\left(-2 \lambda^{2}+2 a \lambda^{2}+a^{2} \lambda^{2}+4 \lambda \mu+2 a \lambda \mu+\mu^{2}\right) x^{2}+2 \lambda(a \lambda+\mu) x^{3}+\lambda^{2} x^{4}\right) .
\end{aligned}
$$

Therefore

$$
\Delta^{2}\left(F_{\lambda, \mu}\right)=\lambda^{16} \mu^{7}(\lambda(a-1)+\mu)^{2}\left((-2+a)^{3} \lambda^{2}-\left(1-10 a-2 a^{2}\right) \lambda \mu+a \mu^{2}\right) .
$$


Hence the special values $\lambda, \mu$ are

$$
\lambda=0, \quad \mu=0, \quad \mu=(1-a) \lambda, \quad \mu=\frac{1-10 a-2 a^{2} \pm(1+4 a)^{3 / 2}}{2 a} \lambda .
$$

The last three special values correspond to the values

$$
h=0, \quad h=a-1, \quad h=\frac{2 a^{2}+10 a-1 \pm(1+4 a)^{3 / 2}}{2 a},
$$

for which $\mathcal{V}_{a, h}$ is not an elliptic curve. More concretely, it is formed by three straight lines; a straight line and a parabola; and a rational cubic with one real isolated singularity, respectively. These results coincide with the ones presented in [16].

3.2. Bifurcation diagrams of rationally integrable differential systems. We consider in this section polynomial differential systems having a rational (including polynomial) first integral $H=f / g$. It is clear that different coefficients of the system (and hence different coefficients of $H$ ) may provide different behaviors in the phase portrait. So it is important to know the relations among these coefficients to understand the bifurcation diagram of the systems. This can be done using Corollary 2. The level sets of $H, \lambda f+\mu g=0$, provide the (algebraic) solutions of the system; we set $F_{\lambda, \mu}(x, y)=\lambda f+\mu g$. Then $\Delta^{2}\left(F_{\lambda, \mu}\right)$ is a polynomial in $\lambda, \mu$ and in the coefficients of $H$. Afterwards we can apply the resultant with respect to $\lambda$ or $\mu$ to this polynomial and look for the zeroes of the new polynomial to obtain relations among the coefficients of $H$. These relations provide the curves in the coefficients space that may separate different phase portraits, that is curves in the bifurcation diagram of the differential system.

In the sequel we provide three examples of application. More examples of families where our approach can be used can be found for instance in [15, 17, 20, 21].

Example 3. The phase portraits of the quadratic Hamiltonian differential systems are classified in [4]. Several normal forms for quadratic Hamiltonian systems are provided, and some conditions are found for each one of them in order to distinguish different phase portraits. Here we show one of the results about these normal forms.

Proposition 3. Consider the quadratic Hamiltonian system $\dot{x}=b x+c y, \dot{y}=-a x-b y-x^{2}$, with the Hamiltonian $H=a x^{2} / 2+b x y+x^{3} / 3+c y^{2} / 2$. The following cases distinguish among different phase portraits:

(i) $c=0, b=0, a=0$.

(ii) $c=0, b=0, a \neq 0$.

(iii) $c=0, b \neq 0$.

(iv) $c \neq 0, b^{2}-a c=0$.

(v) $c \neq 0, b^{2}-a c \neq 0$.

So for each value of $h$, the level set $H=h$ may have one of the five different behaviors arising from the proposition. The paper considers the finite and infinite singular points that the system may have varying its parameters. Here we arrive to the same result using Corollary 2.

Set $F_{h}=F_{1, h}=H-h=a x^{2} / 2+b x y+x^{3} / 3+c y^{2} / 2-h$. Then, $\Delta_{x}\left(F_{h}\right)=6 h\left(-a^{3}+6 h\right)+36 a b h y+\left(-3 a^{2} b^{2}+3 a^{3} c-36 c h\right) y^{2}+2 b\left(8 b^{2}-9 a c\right) y^{3}+9 c^{2} y^{4}$, $\Delta_{y}\left(F_{h}\right)=-6 c^{2} h+3 c\left(-b^{2}+a c\right) x^{2}+2 c^{2} x^{3}$.

We notice that if $c=0$ then $\Delta_{y}\left(F_{h}\right)=0$ and the degree of $\Delta_{x}\left(F_{h}\right)$ decreases; we shall need to consider this case later on. The double resultants are

$$
\begin{aligned}
& \Delta_{x, y}\left(F_{h}\right)=c^{2} h\left(a^{3}\left(3 a c-4 b^{2}\right)-96 b^{2} h\right)^{3}\left(\left(b^{2}-a c\right)^{3}+6 c^{3} h\right), \\
& \Delta_{y, x}\left(F_{h}\right)=c^{7} h\left(\left(b^{2}-a c\right)^{3}+6 c^{3} h\right) .
\end{aligned}
$$


Hence

$$
\Delta^{2}\left(F_{h}\right)=c^{2} h\left(\left(b^{2}-a c\right)^{3}+6 c^{3} h\right) .
$$

If $c=0$ then $\operatorname{deg}_{y}\left(F_{h}\right)=1$, hence we apply a Tame change of variables $x \mapsto x+y^{2}$ to $F_{h}$ to increase this degree. Now we proceed in the same way as above and we obtain $\Delta^{2}\left(F_{h}\right)=b^{9} h$. If $c=b=0$ then $\dot{x}=0$. Indeed we have $F_{h}=(a / 2+x / 3) x^{2}-h$ and, since $F_{h}$ does not depend on $y$, we compute only the resultant of $F_{h}$ with respect to $x$ : $\Delta_{x}\left(F_{h}\right)=\left(a^{3}-6 h\right) h$.

Next we compute the resultant of $\Delta^{2}\left(F_{h}\right)$ with respect to $h$ in order to obtain the relations among the parameters for which the behaviors change. If $b=c=0$, then the resultant of $\Delta_{x}\left(F_{h}\right)$ with respect to $h$ is $a^{6}$. Indeed, if $a=0$ then $h=0$ is a double zero of $\Delta_{x}\left(F_{h}\right)$ and if $a \neq 0$ then we have two different zeroes of $\Delta_{x}\left(F_{h}\right)$ for $h$. We achieve cases (i) and (ii) of the proposition.

If $c=0$ and $b \neq 0$ then $\Delta^{2}\left(F_{h}\right)$ is linear in $h$, so we do not need to compute the resultant. This is case (iii). If $c \neq 0$ then $\Delta^{2}\left(F_{h}\right)$ is quadratic in $h$, as we showed above. Its resultant with respect to $h$ is $c^{9}\left(b^{2}-a c\right)^{6}$. Indeed if $b^{2}-a c=0$ then $h=0$ is a double zero and if $b^{2}-a c \neq 0$ then $\Delta^{2}\left(F_{h}\right)$ has two different zeroes. We obtain cases (iv) and (v).

All the cases of Proposition 3 (and no others) are found with our method. In short, applying our approach we know the bifurcation diagram of the studied system, providing an alternative proof to that of [4].

Example 4. The cubic differential systems of Lotka-Volterra type having a rational first integral of degree 2 can be written as

$$
\dot{x}=x\left(a+b x+d x^{2}-e y^{2}\right), \quad \dot{y}=y\left(-a-c y+d x^{2}-e y^{2}\right) .
$$

The associated rational first integral is

$$
H=\frac{a+b x+c y+d x^{2}+e y^{2}}{x y} .
$$

In [7], a classification into 16 normal forms is done in order to obtain the 28 topologically non-equivalent phase portrait that these systems have. In all of these normal forms, we have $d, e \in\{-1,0,1\}$ and $a, b, c \geq 0$. For each system, some relations among the coefficients are given. These conditions are related to distinguish whether $a, d, e, b^{2}-4 a d, c^{2}-4 a e$ and $b^{2} e-c^{2} d$ are either positive, or negative, or zero.

We can obtain all these cases with our method. Let $F_{\lambda, \mu}=\lambda\left(a+b x+c y+d x^{2}+e y^{2}\right)+\mu x y$. We compute its resultants:

$$
\begin{aligned}
& \Delta_{x}\left(F_{\lambda, \mu}\right)=d \lambda\left(\left(b^{2}-4 a d\right) \lambda^{2}-2 \lambda(2 c d \lambda+b \mu) y-\left(4 d e \lambda^{2}-\mu^{2}\right) y^{2}\right), \\
& \Delta_{y}\left(F_{\lambda, \mu}\right)=e \lambda\left(\left(c^{2}-4 a e\right) \lambda^{2}-2 \lambda(2 b e \lambda+c \mu) x-\left(4 d e \lambda^{2}-\mu^{2}\right) x^{2}\right) .
\end{aligned}
$$

The double resultants are

$$
\begin{aligned}
& \Delta_{x, y}\left(F_{\lambda, \mu}\right)=d^{4} \lambda^{5}\left(4 d e \lambda^{2}-\mu^{2}\right)\left(\left(c^{2} d+b^{2} e-4 a d e\right) \lambda^{2}+b c \lambda \mu+a \mu^{2}\right), \\
& \Delta_{y, x}\left(F_{\lambda, \mu}\right)=e^{4} \lambda^{5}\left(4 d e \lambda^{2}-\mu^{2}\right)\left(\left(c^{2} d+b^{2} e-4 a d e\right) \lambda^{2}+b c \lambda \mu+a \mu^{2}\right) .
\end{aligned}
$$

Hence

$$
\Delta^{2}\left(F_{\lambda, \mu}\right)=\lambda^{5}\left(4 d e \lambda^{2}-\mu^{2}\right)\left(\left(c^{2} d+b^{2} e-4 a d e\right) \lambda^{2}+b c \lambda \mu+a \mu^{2}\right) .
$$

We note that if $a=0$ then the exponent of $\lambda$ in $\Delta^{2}\left(F_{\lambda, \mu}\right)$ increases, and if $d e=0$ then $\mu$ is a double factor. So we first distinguish whether $a d e \neq 0$ or not. We notice that we shall apply Tame changes of variables to $F_{\lambda, \mu}$ in some cases for which the degree of $x$ or $y$ is equal to one.

If $a d e \neq 0$, then the resultant of $\Delta^{2}\left(F_{\lambda, \mu}\right)$ with respect to $\mu$ is ade $\left(b^{2}-4 a d\right)\left(c^{2}-4 a e\right)\left(b^{2} e-\right.$ $\left.c^{2} d\right)^{4}$. This case covers completely the families 1,2,3 and 4 of $[7]$ and their phase portraits by distinguishing whether each factor is zero or not. 
If $a \neq 0$ but $d e=0$, then (applying linear changes of variables and time if needed) we can set $a=1, e=0$ and $d^{2}=1$. We have

$$
\Delta^{2}\left(F_{\lambda, \mu}\right)=\lambda^{5} \mu^{2}\left(c^{2} d \lambda^{2}+b c \lambda \mu+\mu^{2}\right) .
$$

The resultant of $\Delta^{2}\left(F_{\lambda, \mu}\right)$ with respect to $\mu$ is $c^{6}\left(b^{2}-4 d\right)$. Since $b, c \geq 0$ and $d^{2}=1$, the special values are $c=0$ and $b=2$, the latter only in the case $d=1$. These special values cover the phase portraits of all families 5 to 10 of [7].

If $a=0$ and $d e \neq 0$, then we can set $e=-1$. We have

$$
\Delta^{2}\left(F_{\lambda, \mu}\right)=\lambda^{6}\left(\left(b^{2}-c^{2} d\right) \lambda-b c \mu\right)\left(4 d \lambda^{2}+\mu^{2}\right) .
$$

The resultant of $\Delta^{2}\left(F_{\lambda, \mu}\right)$ with respect to $\mu$ tells us that the special values are $b=0, c=0$ and $b^{2}+c^{2} d=0$; the latter reads $b-c=0$ and runs only for $d=-1$. The phase portraits of families $11, \ldots, 14$ of $[7]$ cover these situations.

If $a=d e=0$ then we can set $c, d=1$ and $e=0$. We get

$$
\Delta^{2}\left(F_{\lambda, \mu}\right)=\lambda^{6} \mu^{2}(b \lambda+\mu) .
$$

We obtain only the special value $b=0$. We arrive to the families 15 and 16 of [7] depending on whether $b \neq 0$ or $b=0$.

Example 5. A family of cubic Hamiltonian centers is studied in [13]. The 11 nontopologically equivalent phase portraits are classified. The Hamiltonian function is

$$
H(x, y)=\frac{1}{2}\left(\left(x+a x^{2}+b x y+c y^{2}\right)^{2}+y^{2}\right),
$$

with $a^{2}+b^{2}+c^{2} \neq 0$. We can apply the modification of Corollary 2 to obtain the different sets of values of $a, b, c$ that provide the 11 different phase portraits. Let $F_{h}=\left(x+a x^{2}+\right.$ $\left.b x y+c y^{2}\right)^{2}+y^{2}-h$. First we compute the resultants of $F_{h}$ :

$$
\begin{aligned}
\Delta_{x}\left(F_{h}\right)= & a^{6}\left(h-y^{2}\right)^{2}\left(16 a^{2} h-1-4 b y\right. \\
& \left.-2\left(8 a^{2}+3 b^{2}-4 a c\right) y^{2}-4 b\left(b^{2}-4 a c\right) y^{3}-\left(b^{2}-4 a c\right)^{2} y^{4}\right), \\
\Delta_{y}\left(F_{h}\right)= & c^{4} P_{8}(x),
\end{aligned}
$$

where $P_{8}$ is a polynomial of degree 8 in $x$ that depends on $a, b, c, h$. The double resultants are

$$
\begin{aligned}
\Delta_{y}\left(\sqrt{\Delta_{x}\left(F_{h}\right)}\right) & =a^{72}\left(b^{2}-4 a c\right)^{4} h\left(1-2\left(b^{2}+4 a c\right) h+\left(b^{2}-4 a c\right)^{2} h^{2}\right)^{4} P_{3}(h), \\
\Delta_{y, x}\left(F_{h}\right) & =a^{4} b^{4} c^{62}\left(b^{2}-4 a c\right)^{4} h^{2}\left(1+4 c^{2} h\right)^{2} P_{3}(h) P_{6}(h)^{3},
\end{aligned}
$$

where $P_{3}(h)=-a^{2}-b^{2}+2 a c-c^{2}+\left(16 a^{4}+20 a^{2} b^{2}+3 b^{4}-32 a^{3} c-2 a b^{2} c+8 a^{2} c^{2}+2 b^{2} c^{2}+\right.$ $\left.8 a c^{3}\right) h+\left(-b^{2}+4 a c\right)^{2}\left(8 a^{2}-3 b^{2}-8 a c-c^{2}\right) h^{2}+\left(b^{2}-4 a c\right)^{4} h^{3}$ and $P_{6}(h)$ is a polynomial of degree 6 in $h$. Hence

$$
\Delta_{*}^{2}\left(F_{h}\right)=a^{4}\left(b^{2}-4 a c\right)^{4} h P_{3}(h) .
$$

Now the resultant of $\Delta_{*}^{2}\left(F_{h}\right)$ with respect to $h$ is

$$
a^{29} b^{2}\left(b^{2}-4 a c\right)^{36}\left((a-c)^{2}+b^{2}\right)^{2}\left(27 a b^{2}-4(c-2 a)^{3}\right)^{3} .
$$

By distinguishing whether this polynomial vanishes or not we obtain the different phase portraits of the system. 
3.3. Remarkable values of rational first integrals. The remarkable values and remarkable curves of rational first integrals of planar differential systems were first introduced by Poincaré in [23], and afterwards studied by several authors, see [8, 10, 11]. It has been shown in the literature that the remarkable curves play an important role in the phase portrait as they are strongly related to its separatrices. It is proved in [8] that there are a finite number of them. In $[8,11]$ they are related with the inverse integrating factor.

Consider the complex planar polynomial differential system of degree $d \in \mathbb{N}$

$$
\dot{x}=P(x, y), \dot{y}=Q(x, y),
$$

where $P, Q \in \mathbb{C}[x, y]$ are coprime and $d=\max \{\operatorname{deg} P, \operatorname{deg} Q\}$. Suppose that system (4) has a minimal rational first integral $H=f / g$. According to Poincaré [23], the remarkable values are defined as level sets $(\lambda: \mu) \in \mathbb{C P}^{1}$ of $H$ for which the polynomial $F_{\lambda, \mu}(x, y)=$ $\lambda f+\mu g$ factorizes into polynomials of degree lower than $\operatorname{deg} H=\max \{\operatorname{deg} f, \operatorname{deg} g\}$. These polynomials provide the remarkable curves associated to $(\lambda, \mu)$.

If in this factorization some factor has exponent greater than one, then the corresponding remarkable curve and remarkable value are said to be critical. The critical remarkable values were used in [12] to obtain some properties of differential systems having a rational first integral.

The set of values $(\lambda: \mu)$ that vanish $\Delta^{2}\left(F_{\lambda, \mu}\right)$ contains the remarkable values of $H$, because their zeroes are the values for which the topology of the level set of $H$ changes. We provide some examples to illustrate this fact.

Example 6. Consider the polynomial differential system

$$
\dot{x}=-7 x^{3}-y^{3}+4 x y^{3}, \quad \dot{y}=-2 y\left(3 x^{2}-2 y^{3}\right) .
$$

This system has the rational first integral

$$
H=\frac{-x^{6}-2 x^{3} y^{3}+x^{4} y^{3}-y^{6}+4 x y^{6}}{y^{7}},
$$

hence its level sets are given by the zero set of

$$
F_{\lambda, \mu}(x, y)=\lambda\left(-x^{6}-2 x^{3} y^{3}+x^{4} y^{3}-y^{6}+4 x y^{6}\right)+\mu y^{7} .
$$

The resultants of $F_{\lambda, \mu}$ are

$$
\begin{aligned}
\Delta_{x}\left(F_{\lambda, \mu}\right)= & \lambda^{6}(3 \lambda-\mu)\left(9 \lambda^{2}+3 \lambda \mu+\mu^{2}\right) y^{33}\left(729 \lambda^{2}-1458 \lambda \mu y+729 \mu^{2} y^{2}\right. \\
& \left.-3672 \lambda^{2} y^{3}+216 \lambda \mu y^{4}+16 \lambda^{2} y^{6}\right), \\
\Delta_{y}\left(F_{\lambda, \mu}\right)= & \lambda^{6} \mu(3 \lambda-\mu)\left(9 \lambda^{2}+3 \lambda \mu+\mu^{2}\right) x^{33}\left(46656 \lambda^{3}-734832 \lambda^{3} x\right. \\
& +4293324 \lambda^{3} x^{2}-7\left(1548531 \lambda^{3}-117649 \mu^{3}\right) x^{3} \\
& \left.+9050832 \lambda^{3} x^{4}+2739744 \lambda^{3} x^{5}+241920 \lambda^{3} x^{6}+6912 \lambda^{3} x^{7}\right) .
\end{aligned}
$$

Moreover,

$$
\begin{aligned}
& \Delta_{x}\left(\sqrt{\Delta_{y}\left(F_{\lambda, \mu}\right)}\right)=\lambda^{114} \mu^{30}(3 \lambda-\mu)^{17}\left(9 \lambda^{2}+3 \lambda \mu+\mu^{2}\right)^{17}, \\
& \Delta_{y}\left(\sqrt{\Delta_{x}\left(F_{\lambda, \mu}\right)}\right)=\lambda^{98}(3 \lambda-\mu)^{15}\left(9 \lambda^{2}+3 \lambda \mu+\mu^{2}\right)^{15} .
\end{aligned}
$$

Therefore

$$
\Delta_{*}^{2}\left(F_{\lambda, \mu}\right)=\lambda^{98}(3 \lambda-\mu)^{15}\left(9 \lambda^{2}+3 \lambda \mu+\mu^{2}\right)^{15} .
$$

The zeroes of $\Delta_{*}^{2}\left(F_{\lambda, \mu}\right)$ are

$$
\lambda=0, \quad \lambda=\frac{\mu}{3}, \quad \lambda=-\frac{1+\sqrt{3} i}{6} \mu, \quad \lambda=-\frac{1-\sqrt{3} i}{6} \mu,
$$

which are exactly the (critical) remarkable values of $H$. 
Example 7. The rational function

$$
H=\frac{(x-2 y)^{3}}{\left(x^{2}-1\right) y}
$$

is a first integral of the cubic polynomial differential system

$$
\dot{x}=\left(x^{2}-1\right)(x+4 y), \quad \dot{y}=y\left(-3+x^{2}+4 x y\right) .
$$

The only remarkable values of $H$ are $\lambda=0$ and $\mu=0$. This can be proved by solving the equation

$$
F_{\lambda, \mu}=\lambda(x-2 y)^{3}+\mu\left(x^{2}-1\right) y=P_{1}(x, y) P_{2}(x, y),
$$

where $P_{i}$ are polynomials of degree $i, i=1,2$.

Let us see how to obtain the same result using our approach. The resultants of $F_{\lambda, \mu}$ are

$$
\begin{aligned}
& \Delta_{x}\left(F_{\lambda, \mu}\right)=\lambda \mu^{2} y^{2}\left(27 \lambda^{2}-4\left(54 \lambda^{2}-18 \lambda \mu+\mu^{2}\right) y^{2}+16 \lambda(27 \lambda-2 \mu) y^{4}\right), \\
& \Delta_{y}\left(F_{\lambda, \mu}\right)=\lambda^{2} \mu^{2}\left(x^{2}-1\right)^{2}\left(2 \mu+(27 \lambda-2 \mu) x^{2}\right) .
\end{aligned}
$$

Hence

$$
\begin{aligned}
& \Delta_{y}\left(\sqrt{\Delta_{x}\left(F_{\lambda, \mu}\right)}\right)=\lambda^{17} \mu^{20}(\mu-12 \lambda)^{6}(27 \lambda-2 \mu)^{2}, \\
& \Delta_{x}\left(\sqrt{\Delta_{y}\left(F_{\lambda, \mu}\right)}\right)=\lambda^{18} \mu^{15}(27 \lambda-2 \mu)^{2} .
\end{aligned}
$$

Thus $\Delta_{*}^{2}\left(F_{\lambda, \mu}\right)=\lambda^{17} \mu^{15}(27 \lambda-2 \mu)^{2}$. The remarkable values $\lambda=0$ and $\mu=0$ are zeroes of this double resultant. There is the extra special value $\mu / \lambda=27 / 2$, which is not a remarkable value. It can be seen that this level set provides an isolated singular point, which is a center at infinity for system (5). If $\mu / \lambda>27 / 2$ then the level sets are periodic orbits surrounding the center. If $\mu / \lambda<27 / 2$ then the level sets are not real curves. The value $27 / 2$ is not a remarkable value of the first integral, but there is a change in the topology of the curves, so it must appear in $\Delta_{*}^{2}\left(F_{\lambda, \mu}\right)$.

This example also shows that the remarkable values are special values of the double resultant; but also that there may exist special values of the double resultant which are not remarkable values of $H$.

3.4. The different topologies of offset curves. In this section, we show an application of the double resultant to detect changes on the topological behavior of offset curves of rationally parametrized curves. The notion of offset is directly related to the concept of envelope. More precisely, the offset curve, at distance $d$, to an irreducible curve $C$ over $C$ is "essentially" the envelope of the system of circles centered at the points of $C$ with fixed radius $d$. A more formal description of offset varieties can be found in [2].

The study of offsets is an active research area. Indeed, as a consequence of this research, many interesting questions related to algebraic geometry, such as the study of the unirationality of the components of the offset, or the construction of rational parametrizations of the components of an offset, have been treated.

Consider a rationally parametrized curve $C:(x(t), y(t))$. Since $\frac{\left(-y^{\prime}(t), x^{\prime}(t)\right)}{\sqrt{x^{\prime}(t)^{2}+y^{\prime}(t)^{2}}}$ is its unit normal vector, the offset curve of $C$ at distance $d$ is

$$
(u(t), v(t))=(x(t), y(t))+d \frac{\left(-y^{\prime}(t), x^{\prime}(t)\right)}{\sqrt{x^{\prime}(t)^{2}+y^{\prime}(t)^{2}}} .
$$

The implicit expression of the offset is typically defined as $F_{d}(u, v)=\operatorname{Res}(P, Q, t)$, where

$$
\begin{aligned}
& P=\operatorname{Num}\left((u-x(t)) \sqrt{x^{\prime}(t)^{2}+y^{\prime}(t)^{2}}+d y^{\prime}(t)\right), \\
& Q=\operatorname{Num}\left((v-y(t)) \sqrt{x^{\prime}(t)^{2}+y^{\prime}(t)^{2}}-d x^{\prime}(t)\right),
\end{aligned}
$$


where $\operatorname{Num}($ ) applied to a rational expression denotes its numerator and it is assumed that the parametrization is such that $\sqrt{x^{\prime}(t)^{2}+y^{\prime}(t)^{2}}$ is again a rational function.

This further property on the parametrization is not always easy to be found but there are some techniques that allow to achieve it, see for example [3]. We introduce here a simple way to characterize the offset that does not need this change of parametrization. Indeed, we define

$$
\begin{aligned}
& R=\operatorname{Num}\left(s^{2}-x^{\prime}(t)^{2}-y^{\prime}(t)^{2}\right), \\
& \widehat{P}=\operatorname{Num}\left((u-x(t)) s+d y^{\prime}(t)\right), \\
& \widehat{Q}=\operatorname{Num}\left((v-y(t)) s-d x^{\prime}(t)\right) .
\end{aligned}
$$

Afterwards we compute $E_{1}=\operatorname{Res}(\widehat{P}, \widehat{Q}, s)$ and $E_{2}=\operatorname{Res}(\widehat{P}, R, s)$. These computations remove the square root from our expressions. Finally we define the offset as

$$
F_{d}(u, v)=\operatorname{Res}^{\prime}\left(\frac{E_{1}}{\operatorname{gcd}\left(E_{1}, E_{2}\right)}, \frac{E_{2}}{\operatorname{gcd}\left(E_{1}, E_{2}\right)}, t\right),
$$

where we compute the gcd respect to the variable $t$ and $\operatorname{Res}^{\prime}()$ denotes first the usual resultant and afterwards the elimination of the trivial factor $u^{n} v^{m}, n, m \in \mathbb{N}$, in case that it appears. Then we can use our approach to find the special values of $F_{d}$. We see some examples next.

Example 8. Consider the parametrized curve $\left(t^{4}, t^{5}\right)$. To compute its offset we first compute the expressions

$$
\widehat{P}=5 d t^{4}-S t^{4}+s u, \quad \widehat{Q}=-4 d t^{3}-S t^{5}+s v, \quad R=s^{2}-16 t^{6}-25 t^{8} .
$$

Next we have

$$
\begin{aligned}
& E_{1}=\operatorname{Res}(\widehat{P}, \widehat{Q}, s)=d t^{3}\left(4 t^{4}+5 t^{6}-4 u-5 t v\right) \\
& E_{2}=\operatorname{Res}(\widehat{P}, R, s)=t^{6}\left(25 d^{2} t^{2}-16 t^{8}-25 t^{10}+32 t^{4} u+50 t^{6} u-16 u^{2}-25 t^{2} u^{2}\right) .
\end{aligned}
$$

Then, $\operatorname{gcd}\left(E_{1}, E_{2}\right)=t^{3}$ and in this case

$$
F_{d}=\operatorname{Res}^{\prime}\left(\frac{E_{1}}{t^{3}}, \frac{E_{2}}{t^{3}}\right)=\frac{1}{u^{5}} \operatorname{Res}\left(\frac{E_{1}}{t^{3}}, \frac{E_{2}}{t^{3}}\right) .
$$

The expression of $F_{d}(u, v)$ has degree 12 in $u$ and 10 in $v$. Its total degree is 12 . There are 25 values of $d$ that vanish $\Delta_{*}^{2}\left(F_{d}\right)$. Only three of them are real: 0 and $\pm 2 /(25 \sqrt{5})$. Since $d \geq 0$, in order to study the different topologies of this offset we only need to distinguish the cases $0<d<2 /(25 \sqrt{5}), d=2 /(25 \sqrt{5})$ and $d>2 /(25 \sqrt{5})$.

In [2] the double discriminant is used to study the topology of the offset curves, see also [1]. Our approach using the double resultant $\Delta_{*}^{2}\left(F_{d}\right)$ is more precise in the sense that it discards some superfluous cases, because of the computation of both double resultants. We next show an example.

Example 9. Consider the parametric curve

$$
\begin{aligned}
& u=\frac{57 t-59 t d+45 d^{2}-8 t^{3}-93 t d^{2}+92 t^{2} d^{2}}{-18 t+31 t^{2}-26 t d-62 t^{3}+t^{2} d^{2}-47 d^{4}} \\
& v=\frac{-1+94 t^{2}+83 d^{2}-86 t d^{2}+23 d^{3}-84 t^{3} d}{-18 t+31 t^{2}-26 t d-62 t^{3}+t^{2} d^{2}-47 d^{4}}
\end{aligned}
$$

provided in [1, Family 12]. The algorithms used there give either 23 or 30 real special values, depending on the algorithm. From our approach we only obtain 6 values: $d \approx-0.15384$, $d \approx-0.12926, d \approx 0.14419, d \approx 0.59722, d \approx 1104.27468$ and $d \approx-1104.26873$. Hence most of them are superfluous. We do not include the computations for the sake of simplicity. 


\section{Some Remarks about the Modified Method}

A first point to reflect is what do the resultants of $F_{b}$ compute. When applying the resultant to $F_{b}$ with respect to $y$, linear factors of the form $x-x_{i}(b)$ of $\Delta_{y}\left(F_{b}\right)$ appear because of values $\left(x_{i}, y_{i}\right)$ for which $F_{b}\left(x_{i}, y_{i}\right)=\frac{\partial F_{b}}{\partial y}\left(x_{i}, y_{i}\right)=0$; that is, points on which the gradient of $F_{b}$ is horizontal. The multiplicity of such factors is related to the number of values $y_{i}$ for which this happens, counting also the corresponding multiplicities.

The second resultant $\Delta_{y, x}\left(F_{b}\right)$ vanishes if and only if either $\Delta_{y}\left(F_{b}\right)$ has some multiple factor or its degree decreases. The first situation happens if either there are (more than one) points $\left(x_{i}, y_{i}\right)$ for which the gradient of $F_{b}$ is horizontal, or there is a singular point on $x=x_{i}$. We notice that with the double resultant $\Delta_{y, x}\left(F_{b}\right)$ we cannot know about a situation where two points on $x=x_{i}$ collide for a special value $b^{*}$, because $\Delta_{y, x}\left(F_{b}\right)=0$ for all $b$, but after a rotation the double straight line becomes two different straight lines that collide for $b^{*}$, and in particular $\Delta_{y, x}\left(F_{b^{*}}\right)=0$.

Recall also that when applying the discriminant or the resultant to a polynomial $F$, it may happen that $F$ has a multiple factor. In this case, both $F$ and its derivative have this factor in common, so the discriminant and the resultant must be zero. These facts may cause problems when computing iterated discriminant or resultants, since if at some step the described situation appears then no information will be obtained.

The way to proceed in the papers using iterated discriminants is to remove this multiplicity and to compute in the second step the discriminant of the "squared-free" polynomial $\sqrt{\operatorname{disc}_{y}\left(F_{b}\right)}$ that is no more identically zero.

Our proposal of computing $\Delta_{*}^{2}\left(F_{b}\right)$ instead of $\Delta^{2}\left(F_{b}\right)$ goes precisely in the same direction described above. Next we want to give an approach of why we believe that this argument works and no special values are omitted. We recall that we are assuming that the polynomial $F_{b}$ is irreducible. Hence a multiple factor cannot appear when computing the first resultant. So we deal with $\Delta_{y}\left(F_{b}\right)$ (the study for $\Delta_{x}\left(F_{b}\right)$ follows in the same way).

Since $\Delta_{y}\left(F_{b}\right)$ is a polynomial in $x$, we can write

$$
\Delta_{y}\left(F_{b}\right)=\delta_{y}(b) \prod_{i=1}^{N}\left(x-x_{i}(b)\right)^{\alpha_{i}},
$$

where $N \in \mathbb{N}, \delta_{y}, x_{i} \in \mathbb{C}[b]$ and $\alpha_{i} \in \mathbb{N}$, for all $i \in\{1, \ldots, N\}$. Suppose that there exists $1 \leq i \leq N$ such that $\alpha_{i}>1$. We distinguish some cases. Suppose first that $\left(x-x_{i}(b)\right)^{\alpha_{i}} \mid \Delta_{y}\left(F_{b}\right)$ for a finite number of values of $b$. Take one of them, say $b^{*}$. There are some extra possibilities:

(1) If for some values $y_{i}^{j}\left(b^{*}\right)$ we have $F_{b^{*}}\left(x_{i}\left(b^{*}\right), y_{i}^{j}\left(b^{*}\right)\right)=\frac{\partial F_{b^{*}}}{\partial y}\left(x_{i}\left(b^{*}\right), y_{i}^{j}\left(b^{*}\right)\right)=0$, $j \in\{1, \ldots, M\}, M \in \mathbb{N} \backslash\{1\}$, then we can rotate $F_{b}$. So this case can be avoided.

(2) If for only one value $y_{i}\left(b^{*}\right)$ we have $F_{b^{*}}\left(x_{i}\left(b^{*}\right), y_{i}\left(b^{*}\right)\right)=\frac{\partial F_{b^{*}}}{\partial y}\left(x_{i}\left(b^{*}\right), y_{i}\left(b^{*}\right)\right)=0$, then this value remains for any rotation applied to $F_{b}$, in particular a $\pi / 2$ rotation. Hence $\frac{\partial F_{b^{*}}}{\partial x}\left(x_{i}, y_{i}\right)=0$ and therefore $\left(x_{i}, y_{i}\right)$ is a singular point of $F_{b^{*}}$. Thus by Theorem 1 we have $\Delta^{2}\left(F_{b^{*}}\right)=0$.

In the former case, and following the arguments above, the multiplicity $\alpha_{i}$ comes from the collision of $\alpha_{i}>1$ straight lines at the value $b^{*}$. Of course this behavior is detected by the resultant of $\Delta_{y}\left(F_{b}\right)$ and thus the value $b^{*}$ appears in the factorization of $\Delta_{y, x}\left(F_{b}\right)$.

Next we consider the case that $\left(x-x_{i}\right)^{\alpha_{i}} \mid \Delta_{y}\left(F_{b}\right)$ for all $b$. Following the previous arguments it is clear that $F_{b}$ has a singular point at $x=x_{i}$, and this happens for all $b$. Hence $\Delta_{y, x}\left(F_{b}\right)=0$ by Theorem 1 .

After these arguments we can assume that special values are associated to changes in the multiplicity of the straight lines of $\Delta_{y}\left(F_{b}\right)$. Therefore we may consider $\sqrt{\Delta_{y}\left(F_{b}\right)}$ to 
compute the double resultant, and from this square-free polynomial we still obtain all the special values of $F_{b}$.

\section{REFERENCES}

[1] J.G. AlCÁzAR, On the different shapes arising in a family of plane rational curves depending on a parameter, Comput. Aided Geom. D. 27 (2010), 162-178.

[2] J.G. Alcázar, J. Schicho and J.R. Sendra, A delineability-based method for computing critical sets of algebraic surfaces, J. Symb. Comput. 42 (2007), 678-691.

[3] E. Arrondo, J. Sendra And J.R. Sendra, Parametric Generalized Offsets to Hypersurfaces, J. Symb. Comput. 23 (1997), 267-285.

[4] J.C. Artés And J. Llibre, Quadratic Hamiltonian vector fields, J. Diff. Eq. 107 (1994), 80-95.

[5] G. Bastien And M. Rogalski, Global Behavior of the Solutions of Lyness' Difference Equation $u_{n+2} u_{n}=u_{n+1}+a$, J. Difference Equations Appl. 10 (2004), 977-1003.

[6] L. Busé, B. Mourrain, Explicit factors of some iterated resultants and discriminants, Math. Comp. 78 (2009) (265), 345-386.

[7] L. Cairó And J. Llibre, Phase portraits of cubic polynomial vector fields of Lotka-Volterra type having a rational first integral of degree 2, J. Phys. A: Math. Theor. 40 (2007), 6329-6348.

[8] J. Chavarriga, H. Giacomini, J. Giné And J. Llibre, Darboux integrability and the inverse integrating factor, J. Diff. Eq. 194 (2003), 116-139.

[9] D. Cox, J. Little And D. O'Shea, Using algebraic geometry, Springer-Verlag New York, 1998.

[10] A. Ferragut and H. Giacomini, A new algorithm for finding rational first integrals of polynomial vector fields, Qual. Theory Dyn. Syst. 9 (2010), 89-99.

[11] A. Ferragut And J. LliBre, On the remarkable values of the rational first integrals of polynomial vector fields, J. Diff. Eq. 241 (2007), 399-417.

[12] A. Ferragut, J. Llibre And A. Mahdi, Polynomial inverse integrating factors for polynomial vector fields, Discrete Contin. Dyn. Syst. 17 (2007), 387-395.

[13] M.E. Frías-Armenta and J. Llibre, New family of cubic Hamiltonian centers, preprint 2014.

[14] J. D. García-Saldaña, A. Gasull And H. Giacomini, Bifurcation values for a familiy of planar vector fields of degree five, Discrete Contin. Dyn. Syst. 35:2 (2015), 669-701.

[15] A. Gasull, A. Guillamon And V. MaÑosa, Phase portrait of Hamiltonian systems with homogeneous nonlinearities, Nonl. Anal. 42 (2000), 679-707.

[16] A. Gasull, V. Mañosa and X. Xarles, Rational periodic sequences for the Lyness recurrence, Discrete Contin. Dyn. Syst. 32 (2012), 587-604.

[17] A. Guillamon and Ch. Pantazi, Phase portraits of separable Hamiltonian systems, Nonl. Anal. 74 (2011), 4012-4035.

[18] Han, J., DAI, L., XIA, B., Constructing fewer open cells by gcd computation in cad projection, Proc. ISSAC2014 (2014), 240247.

[19] D. Lazard, S. McCallum, Iterated discriminants, J. Symbolic Comput. 44, (2009), 1176-1193.

[20] J. Llibre, A. Mahdi AND N. Vulpe, Phase portraits and invariant straight lines of cubic polynomial vector fields having a quadratic rational first integral, Rocky Mount. J. Math. 41 (2011), 1585-1629.

[21] J. Llibre AND R. Oliveira, Phase portrait of polynomial quadratic vector fields having a first integral of degree 3, Nonl. Anal. 70 (2009), 3549-3560.

[22] J. Pettigrew, J. A. G. Roberts, Characterizing singular curves in parametrized families of biquadratics, J. Phys. A 41, (2008), 115203, 28 pp.

[23] H. Poincaré, Sur l'intégration des équations différentielles du premier ordre et du premier degré I and II, Rendiconti del Circolo Matematico di Palermo 5 (1891), 161-191; 11 (1897), 193-239.

[24] A. VAn Den Essen, Polynomial automorphisms and the Jacobian conjecture, Basel; Boston: Birkhaüser Verlag, 2000.

${ }^{1}$ Institut Universitari de Matemàtiques i Aplicacions de Castelló (IMAC) and Departament de Matemàtiques; Universitat Jaume I, Edifici Ti (ESteC), Av. De Vicent Sos Baynat, s/n, Campus del Riu Sec, 12071 Castelló de la Plana, Spain

2 Instituto de Matemática y Física, Universidad de Talca, Camino Lircay S/N, Campus Norte, Talca, Chile

3 Departament de Matemàtiques, Universitat Autònoma de Barcelona, Edifici C, 08193 Bellaterra, Barcelona, Catalonia-Spain

E-mail address: ferragut@uji.es, johanna@inst-mat.utalca.cl, gasull@mat.uab.cat 